\title{
Fundamental aspects of quantum Brownian motion
}

Peter Hänggi, and Gert-Ludwig Ingold

Citation: Chaos 15, 026105 (2005); doi: 10.1063/1.1853631

View online: https://doi.org/10.1063/1.1853631

View Table of Contents: http://aip.scitation.org/toc/cha/15/2

Published by the American Institute of Physics

\section{Articles you may be interested in}

Introduction: 100years of Brownian motion

Chaos: An Interdisciplinary Journal of Nonlinear Science 15, 026101 (2005); 10.1063/1.1895505

Brownian Motion of a Quantum Oscillator

Journal of Mathematical Physics 2, 407 (1961); 10.1063/1.1703727

Statistical Mechanics of Assemblies of Coupled Oscillators

Journal of Mathematical Physics 6, 504 (1965); 10.1063/1.1704304

Solution of quantum Langevin equation: Approximations, theoretical and numerical aspects

The Journal of Chemical Physics 120, 8960 (2004); 10.1063/1.1711593

On the generalized Langevin equation: Classical and quantum mechanical ${ }^{a}$ )

The Journal of Chemical Physics 82, 2708 (1985); 10.1063/1.448268

Non-Markovian stochastic processes: Colored noise

Chaos: An Interdisciplinary Journal of Nonlinear Science 15, 026107 (2005); 10.1063/1.1860471

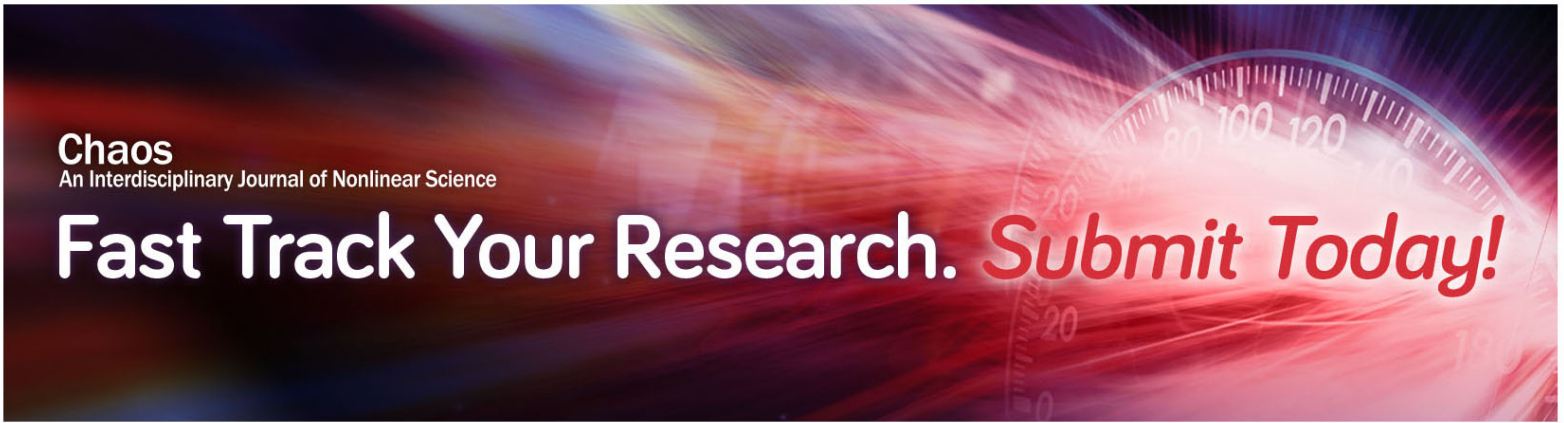




\title{
Fundamental aspects of quantum Brownian motion
}

\author{
Peter Hänggi and Gert-Ludwig Ingold \\ Institut für Physik, Universität Augsburg, 86135 Augsburg, Germany
}

(Received 1 December 2004; accepted 9 December 2004; published online 17 June 2005)

\begin{abstract}
With this work we elaborate on the physics of quantum noise in thermal equilibrium and in stationary nonequilibrium. Starting out from the celebrated quantum fluctuation-dissipation theorem we discuss some important consequences that must hold for open, dissipative quantum systems in thermal equilibrium. The issue of quantum dissipation is exemplified with the fundamental problem of a damped harmonic quantum oscillator. The role of quantum fluctuations is discussed in the context of both, the nonlinear generalized quantum Langevin equation and the path integral approach. We discuss the consequences of the time-reversal symmetry for an open dissipative quantum dynamics and, furthermore, point to a series of subtleties and possible pitfalls. The path integral methodology is applied to the decay of metastable states assisted by quantum Brownian noise. (C) 2005 American Institute of Physics. [DOI: 10.1063/1.1853631]
\end{abstract}

This work deals with the description of quantum Brownian motion in linear and nonlinear quantum systems that exhibit frictional influences. The symmetries of thermal equilibrium impose severe constraints on the time evolution properties of open quantum systems. These lead to a quantum generalization of the classical Einstein relation that connects friction with the strength of thermal quantum fluctuations. There exist a variety of theoretical roadways to model quantum dissipation. Here, we discuss the topic for the prominent case of a damped harmonic oscillator upon combining thermodynamics and linear response theory. A dissipative nonlinear quantum dynamics can be dealt with a generalized quantum Langevin equation, a path integral formulation, or in terms of a generalized quantum master equation for the corresponding reduced dynamics. We illustrate the situation for the problem of the dissipative decay out of a metastable state. Furthermore, we point out a series of subtleties, pitfalls and shortcomings that one must be aware of when confronted with the world of quantum noise driven phenomena.

\section{INTRODUCTION}

Albert Einstein explained the phenomenon of Brownian motion in his annus mirabilis of 1905 by use of statistical methods which he ingeniously combined with the laws of thermodynamics. ${ }^{1}$ In this pioneering work he as well provided a first link between the dissipative forces and the impeding thermal fluctuations, known as the Einstein relation which relates the strength of diffusion to the friction. This intimate connection between dissipation and related fluctuations was put on a firm basis much later when Nyquist ${ }^{2}$ and Johnson $^{3}$ considered the spectral density of voltage- and current-fluctuations.

What role do quantum mechanics and the associated quantum fluctuations play in this context? After the birth of quantum mechanics in the early 1920's we can encounter in the very final paragraph of the 1928 paper by Nyquist for the first time the introduction of quantum mechanical noise via the substitution of the energy $k_{B} T$ from the classical equipartition law ${ }^{4}$ by the thermally averaged quantum energy (but leaving out the zero point energy contribution) of the harmonic oscillator. Nyquist's remark thus constitutes a precursor of the celebrated work by Callen and Welton ${ }^{5}$ who generalized the relations by Einstein, Nyquist, and Johnson to include quantum effects: In their work they put forward a generally valid connection between the response function and the associated quantum fluctuations in equilibrium, the quantum fluctuation-dissipation theorem.

Without doubt, quantum fluctuations constitute a prominent noise source in many nanoscale and biological systems. For example, the tunnelling and the transfer of electrons, quasiparticles, and alike, is assisted by noise for which the quantum nature cannot be neglected. The features of this noise change drastically as a function of temperature: At sufficiently high temperatures a crossover does occur to classical Johnson-Nyquist noise.

With this work we shall present various methods and schemes of modelling quantum Brownian motion from first principles. In particular, the thermal noise must at all times obey the quantum version of the fluctuation-dissipation theorem (à la Callen-Welton). This latter property is necessary in order to be consistent with the second law of thermodynamics and the principle of (quantum) detailed balance. We elaborate on several alternative but equivalent methods to describe quantum noise and quantum Brownian motion per se: These are the functional integral method for dissipative quantum systems ${ }^{6,7}$ and time-dependent driven quantum systems, ${ }^{8}$ the quantum Langevin (operator) approach, ${ }^{9}$ stochastic schemes, ${ }^{10,11}$ or the concept of stochastic Schrödinger equations. ${ }^{12}$ In doing so, we call attention to distinct differences to the classical situation and, as well, identify a series of delicate pitfalls which must be observed when making even innocent looking approximations. Such pitfalls involve, among others, the rotating-wave approximation, the use of quasiclassical Langevin forces, the quantum regression hypothesis and/or the Markov approximation. ${ }^{6,8,13}$ 


\section{THE QUANTUM FLUCTUATION-DISSIPATION THEOREM AND ITS IMPLICATIONS}

As already mentioned, in 1951 Callen and Welton proved a pivotal relation between equilibrium fluctuations and dissipative transport coefficients. Note also that this quantum fluctuation-dissipation relation holds true independent of particle statistics. The following cornerstone achievements can be found in this primary work: ${ }^{5}$

(1) The generalization of the classical Nyquist's formula to the quantum case;

(2) the quantum mechanical proof that susceptibilities are related to the spectral densities of symmetrized correlation functions.

For a single degree of freedom, linear response theory yields for the change of the expectation value of an operatorvalued observable $B$ due to the action of a (classical) force $F(t)$ that couples to the conjugate dynamical operator $A$

$$
\langle\delta B(t)\rangle=\int_{-\infty}^{t} d s \chi_{B A}(t-s) F(s) .
$$

Here, $\delta B(t)=B(t)-\langle B\rangle_{0}$ denotes the difference with respect to the thermal equilibrium average $\langle B\rangle_{0}$ in the absence of the force. The reaction of the system is contained in the response function $\chi_{B A}(t)$ with a so-called dissipative part

$$
\chi_{B A}^{d}(t)=\frac{1}{2 i}\left[\chi_{B A}(t)-\chi_{A B}(-t)\right] .
$$

The Fourier transform of $\chi_{B A}^{d}(t)$ will be denoted by $\widetilde{\chi}_{B A}^{d}(\omega)$. It is worth noting here that only when $A=B$ does this part in fact coincide with the imaginary part of the complex-valued susceptibility $\widetilde{\chi}_{B A}^{\prime \prime}(\omega)$.

The fluctuations are described by the equilibrium correlation function

$$
C_{B A}(t)=\langle\delta B(t) \delta A(0)\rangle_{\beta}
$$

at inverse temperature $\beta=1 / k_{B} T$. The correlation function is complex-valued because the operators $B(t)$ and $A(0)$ in general do not commute. While the antisymmetric part of $C_{B A}(t)$ is directly related to the response function by linear response theory, the power spectrum of the symmetrized correlation function

$$
S_{B A}(t)=\frac{1}{2}\langle\delta B(t) \delta A(0)+\delta A(0) \delta B(t)\rangle
$$

depends on the Fourier transform of the dissipative part of the response function via

$$
S_{B A}(\omega)=\hbar \operatorname{coth}\left(\frac{\hbar \omega}{2 k_{B} T}\right) \widetilde{\chi}_{B A}^{d}(\omega) .
$$

This result is the quantum version of the fluctuationdissipation theorem as it relates the fluctuations described by $S_{B A}(\omega)$ to the dissipative part $\widetilde{\chi}_{B A}^{d}(\omega)$ of the response.

In the spirit of the work by Nyquist and Johnson we consider as an example the response of a current $\delta I$ through an electric circuit subject to a voltage change $\delta V$. This implies $B=I$ and, because the voltage couples to the charge $Q$, $A=Q$. The response of the circuit is determined by $\delta I(\omega)$
$=Y(\omega) \delta V(\omega)$ where the admittance $Y(\omega)$ is identical to the susceptibility $\chi_{I Q}(\omega)$. As a consequence of $I=\dot{Q}$, the symmetrized power spectrum of the current fluctuations is given by $S_{I I}(\omega)=i \omega S_{I Q}(\omega)$ so that we obtain

$$
\begin{aligned}
S_{I I}(\omega) & =\hbar \omega \operatorname{coth}\left(\frac{\hbar \omega}{2 k_{B} T}\right) \operatorname{Re} Y(\omega) \\
& =2\left[\frac{\hbar \omega}{2}+\frac{\hbar \omega}{e^{\beta \hbar \omega}-1}\right] \operatorname{Re} Y(\omega) .
\end{aligned}
$$

In the high temperature limit $k_{B} T \gg \hbar \omega$, we recover the results of Nyquist and Johnson, i.e. $S_{I I}(\omega) \rightarrow 2 k_{B} T \operatorname{Re} Y(\omega)$. For the Markovian limit of an Ohmic resistor, where $Y(\omega)$ $=1 / R$, this result simplifies to read $S_{I I}(\omega)=2 k_{B} T / R$. The quantum version was already anticipated by Nyquist in the last paragraph of his 1928 paper. $^{2}$ However, he made use of the original expression of Planck which yields only the second contribution present in the lower line of (6). Nyquist thus missed the first term arising from the vacuum energy which already appears in a paper by Planck published in $1911 .^{14}$

On the other hand, in the extreme quantum limit $k_{B} T$ $\ll \hbar \omega$, we find that $S_{I I}(\omega) \rightarrow \hbar \omega \operatorname{Re} Y(\omega)$. In particular, this implies that at zero frequency the spectral weight of the current fluctuations vanishes in the generic case where the admittance does not exhibit an infrared divergence.

We cannot emphasize enough that the quantum fluctuation-dissipation relation (5) and corresponding implications hold true for any isolated, closed quantum system. Thus, upon contracting the dynamics in full phase space onto a reduced description of an open quantum system exhibiting dissipation these relations hold true nevertheless. Therefore, care must be taken when invoking approximations in order to avoid any violation of these rigorous relations. We next consider the role of quantum dissipation for an exactly solvable situation: the damped quantum harmonic oscillator dynamics.

\section{QUANTUM DISSIPATION: THE DAMPED HARMONIC OSCILLATOR}

\section{A. Equilibrium correlation functions}

Let us next consider the most fundamental case of a simple open quantum system, namely, the damped harmonic oscillator. This problem could be tackled by setting up a microscopic model describing the coupling to environmental degrees of freedom to which energy can be transferred irreversibly, thus giving rise to dissipation. Such an approach will be introduced in Sec. IV A. On the other hand, the linearity of the damped harmonic oscillator allows us as alternative to proceed on a phenomenological level. This approach is closely related to the usual classical procedure where damping is frequently introduced by adding in the equation of motion a force proportional to the velocity.

Classically, the motion of a harmonic oscillator subject to linear friction is determined by 


$$
M \ddot{q}+M \int_{-\infty}^{t} d s \gamma(t-s) \dot{q}(s)+M \omega_{0}^{2} q=0 .
$$

In the example of an electric circuit mentioned in the previous section, a damping kernel $\gamma(t)$ with memory would correspond to a frequency-dependent admittance. In the special case of ohmic friction corresponding to $Y(\omega)=1 / R$, the damping force is proportional to the velocity of the harmonic oscillator, so that the equation of motion reads

$$
M \ddot{q}+M \gamma \dot{q}+M \omega_{0}^{2} q=0 .
$$

In (7) and (8) the mass, frequency, and position of the oscillator are denoted by $M, \omega_{0}$, and $q$, respectively. Due to the Ehrenfest theorem, the equation of motion (7) is still valid in the quantum regime if we replace $q$ by its expectation value. As a consequence, the quantum mechanical dynamic susceptibility agrees with the classical expression ${ }^{15,16}$

$$
\chi_{q q}(\omega)=\frac{1}{M-\omega^{2}-i \omega \tilde{\gamma}(\omega)+\omega_{0}^{2}},
$$

where $\widetilde{\gamma}(\omega)$ denotes the Fourier transform of the damping kernel $\gamma(t)$.

As mentioned before, the response function directly yields the antisymmetric part of the position autocorrelation function $C_{q q}(t)$. It therefore suffices to discuss the symmetrized part $S_{q q}(t)$ defined according to (4). Furthermore, our linear system with linear damping represents a stationary Gaussian process so that all higher order correlation functions may be expressed in terms of second order correlation functions. ${ }^{15}$ In addition, equilibrium correlation functions containing momentum operators $p$ can be reduced to position correlation functions by means of $p=M \dot{q}$. The dynamics of the damped harmonic oscillator can therefore entirely be described by the response function, i.e., the Fourier transform of (9) and $S_{q q}(t) .6,15,16$

In the case of Ohmic damping, $\widetilde{\gamma}(\omega)=\gamma$, the position autocorrelation can be explicitly evaluated from the fluctuation-dissipation theorem (5). The inverse Fourier transform into the time domain is determined by the poles on the right-hand side. The dissipative part of the dynamic susceptibility leads to four poles at $\omega= \pm(\bar{\omega} \pm i \gamma / 2)$ with $\bar{\omega}$ $=\left(\omega_{0}^{2}-\gamma^{2} / 4\right)^{1 / 2}$ which contribute to the correlation function $S_{q q}(t)$ at all temperatures. At sufficiently low temperatures, the poles of the hyperbolic cotangent at $\omega= \pm i \nu_{n}$ with the Matsubara frequencies $\nu_{n}=2 \pi n / \hbar \beta$ become important as well. After performing the contour integration in (5), one arrives at ${ }^{6,15,16}$

$$
\begin{aligned}
S_{q q}(t)= & \frac{\hbar}{2 M \bar{\omega}} \exp \left(-\frac{\gamma}{2}|t|\right) \\
& \times \frac{\sinh (\hbar \beta \bar{\omega}) \cos (\bar{\omega} t)+\sin (\hbar \beta \gamma / 2) \sin (\bar{\omega}|t|)}{\cosh (\hbar \beta \bar{\omega})-\cos (\hbar \beta \gamma / 2)} \\
& -\frac{2 \gamma}{M \beta} \sum_{n=1}^{\infty} \frac{\nu_{n} \exp \left(-\nu_{n}|t|\right)}{\left(\nu_{n}^{2}+\omega_{0}^{2}\right)^{2}-\gamma^{2} \nu_{n}^{2}}
\end{aligned}
$$

In the limit of high temperatures the second term vanishes and the first term yields the classical correlation func- tion. Quantum corrections to this term are relevant at temperatures of the order $\hbar \omega_{0} / k_{B}$ or below, and these corrections may be obtained from weak coupling theories like the quantum master equation approach. ${ }^{17-20}$ However, there is another regime at temperatures below $\hbar \gamma / 4 \pi k_{B}$. Here, the second term may initially be small, but nevertheless it may dominate the long-time behavior of the correlation function. This becomes particularly apparent in the limit of zero temperature where the exponential functions in the second term in Eq. (10) sum up to an algebraic long-time behavior, i.e., $S_{q q}(t)=-\left(\hbar \gamma / \pi M \omega_{0}^{4}\right) t^{-2}$. Its relevance for the dynamical evolution of the damped harmonic oscillator depends on the details of the initial preparation. ${ }^{21}$ Although the algebraic decay results from the zero temperature limit it can also be observed at low, but finite temperatures during intermediate times before an exponential decay with time constant $\nu_{1}$ sets in. $^{22}$ The occurrence of additional time scales besides $\gamma$ at low temperatures leads to shortcomings with the quantum regression hypothesis and allows for the decay of correlations on time scales longer than $\gamma^{13}$

\section{B. The reduced density matrix and the partition function}

In the previous section, we have seen that the dynamics of a damped harmonic oscillator can be fully described in terms of the position autocorrelation function (10) and its time derivatives as well as the (classical) response function. If one is interested only in equilibrium expectation values of arbitrary operators acting in the Hilbert space of the harmonic oscillator, it is sufficient to know the reduced density matrix. By means of arguments analogous to the dynamic case presented in the previous section, the reduced density matrix can only depend on second moments of position and momentum, $\left\langle q^{2}\right\rangle_{\beta}$ and $\left\langle p^{2}\right\rangle_{\beta}$, respectively. The equilibrium density matrix then necessarily takes the form ${ }^{6}$

$$
\begin{aligned}
\rho_{\beta}\left(q, q^{\prime}\right)= & \frac{1}{\left(2 \pi\left\langle q^{2}\right\rangle\right)^{1 / 2}} \\
& \times \exp \left[-\frac{\left(q+q^{\prime}\right)^{2}}{8\left\langle q^{2}\right\rangle_{\beta}}-\frac{\left\langle p^{2}\right\rangle_{\beta}}{\hbar^{2}}\left(q-q^{\prime}\right)^{2}\right] .
\end{aligned}
$$

The second moments are found to read

$$
\left\langle q^{2}\right\rangle_{\beta}=\frac{1}{M \beta} \sum_{n=-\infty}^{+\infty} \frac{1}{\omega_{0}^{2}+\nu_{n}^{2}+\left|\nu_{n}\right| \hat{\gamma}\left(\left|\nu_{n}\right|\right)}
$$

and

$$
\left\langle p^{2}\right\rangle_{\beta}=\frac{M}{\beta} \sum_{n=-\infty}^{+\infty} \frac{\omega_{0}^{2}+\left|\nu_{n}\right| \hat{\gamma}\left(\left|\nu_{n}\right|\right)}{\omega_{0}^{2}+\nu_{n}^{2}+\left|\nu_{n}\right| \hat{\gamma}\left(\left|\nu_{n}\right|\right)},
$$

where we have introduced the Laplace transform of the damping kernel

$$
\hat{\gamma}(z)=\int_{0}^{\infty} d t \exp (-z t) \gamma(t)
$$

We note that for strictly Ohmic damping the second moment of the momentum (13) exhibits a logarithmic divergence which can be removed by introducing a finite memory 


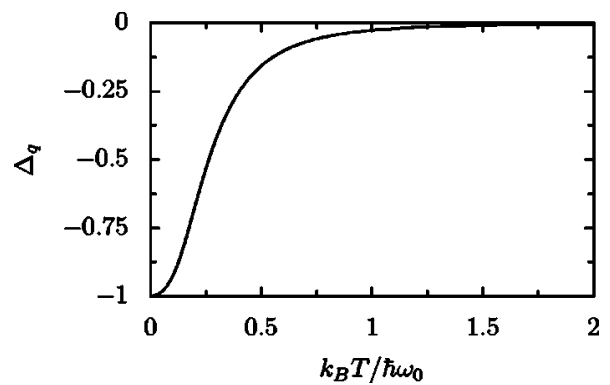

FIG. 1. The weak coupling correction $\Delta_{q}$ to $\left\langle q^{2}\right\rangle_{\beta}$ according to (16) is depicted as a function of the temperature $T$. For $k_{B} T \gg \hbar \omega_{0}$, the correction becomes negligible.

to the damping mechanism. For finite coupling to the environment, i.e., for finite damping strength $\gamma$, the reduced density matrix (11) obviously does not agree with the canonical density matrix $\exp \left(-\beta H_{S}\right)$ at the same temperature, where $H_{S}$ denotes the Hamiltonian of the undamped harmonic oscillator.

In order to get an idea of the deviation of the true reduced density matrix from the canonical one, we consider the leading corrections to the second moment of the position due to the finite coupling to the environment. Expanding in orders of the damping strength $\gamma$, we obtain for Ohmic damping

$$
\frac{\left\langle q^{2}\right\rangle_{\beta}(\gamma)}{\left\langle q^{2}\right\rangle_{\beta}(\gamma=0)}=1+\frac{\gamma}{\pi \omega_{0}} \Delta_{q}+O\left(\gamma^{2}\right)
$$

with

$$
\Delta_{q}=\frac{\hbar \beta \omega_{0}}{2 \pi} \frac{\operatorname{Im} \psi^{\prime}\left(i \frac{\hbar \beta \omega_{0}}{2 \pi}\right)}{\operatorname{coth}\left(\frac{\hbar \beta \omega_{0}}{2}\right)} .
$$

Here, $\psi^{\prime}$ denotes the first derivative of the digamma function. The correction $\Delta_{q}$ is depicted in Fig. 1 as a function of temperature. We find that the leading corrections are particularly important in the quantum regime, $k_{B} T \ll \hbar \omega_{0}$, while in the classical regime the corrections to the canonical density matrix are negligible.

As we have already mentioned, a finite memory time of the damping kernel or, equivalently, a finite cutoff frequency $\omega_{D}$ for the environmental mode spectrum is needed in order to keep the second moment of the momentum (13) finite. If $\omega_{D} \gg \omega_{0}, \gamma$, the corrections to the canonical density matrix for weak coupling will only be small if the temperature is larger than the cutoff frequency, i.e., $k_{B} T \gg \hbar \omega_{D}$.

The differences between the correct reduced density matrix (11) and the canonical one are also reflected in the partition function. Without specifying a microscopic model for the environment, the partition function $\mathcal{Z}$ for the damped harmonic oscillator can be obtained by the requirement that it generates the second moment of position according to ${ }^{15}$

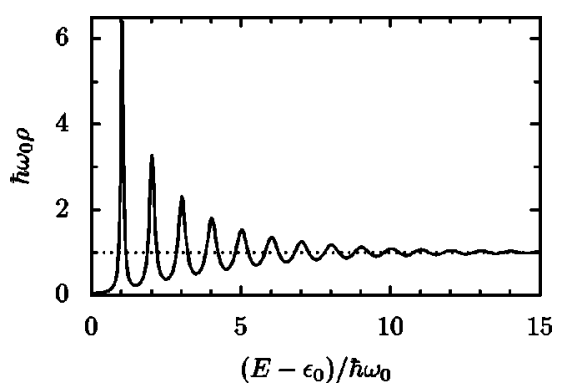

FIG. 2. The density of states defined by inversion of the relation (19) for a weakly damped harmonic oscillator with $\gamma=0.1 \omega_{0}$ exhibits broadened peaks close to the energies $\epsilon_{0}+n \hbar \omega_{0}$. A delta function at the ground state energy $\epsilon_{0}$ is not shown explicitly. The dotted line represents the average density of states.

$$
\left\langle q^{2}\right\rangle_{\beta}=-\frac{1}{M \beta \omega_{0}} \frac{d}{d \omega_{0}} \ln (\mathcal{Z}) .
$$

This leads to the product representation of the partition function, i.e.,

$$
\mathcal{Z}=\frac{1}{\hbar \beta \omega_{0}} \prod_{n=1}^{\infty} \frac{\nu_{n}^{2}}{\nu_{n}^{2}+\nu_{n} \hat{\gamma}\left(\nu_{n}\right)+\omega_{0}^{2}} .
$$

The properties of this partition function become more transparent if one relates it to a density of states $\rho(E)$ according to ${ }^{23}$

$$
\mathcal{Z}(\beta)=\int_{0}^{\infty} d E \rho(E) \exp (-\beta E)
$$

The factor $\left(\hbar \beta \omega_{0}\right)^{-1}$ in (18) can then be interpreted in terms of the average density of states $\left(\hbar \omega_{0}\right)^{-1}$ indicated in Fig. 2 as a dotted line. We further note that the partition function diverges for strict Ohmic damping. However, it can be shown that this divergence is entirely due to a divergence of the ground state energy $\epsilon_{0}$ in the presence of Ohmic dissipation. ${ }^{24}$ In contrast, for a bath with a spectral density possessing a high-frequency cutoff, $\epsilon_{0}$ emerges to be finite [see Eq. (1.191) in Ref. 24]. For large cutoff frequencies, the poles of the partition function, which determine the density of states, can then be determined from the condition $\nu_{n}^{2}$ $+\gamma \nu_{n}+\omega_{0}^{2}=0$. These poles give rise to a density of states which for weak damping exhibits narrow peaks whose width is in agreement with the result from Fermi's Golden Rule. Figure 2 depicts an example for $\gamma / \omega_{0}=0.1$. In view of the remark made before, the density of states is shifted by the ground state energy. In addition, a delta peak at the ground state energy has been omitted. With increasing damping strength, the peaks broaden so that for sufficiently strong damping a rather featureless density of states results which decreases with increasing energy to the average density of states (cf. Fig. 3 in Ref. 23). 


\section{DISSIPATION IN NONLINEAR QUANTUM SYSTEMS: THE GENERALIZED QUANTUM LANGEVIN EQUATION (QLE)}

\section{A. Bath of oscillators}

For nonlinear systems the arguments given in the previous section no longer apply. In particular, second-order correlation functions are not sufficient anymore to completely describe the damped system. An alternative approach to quantum dissipative systems starting from a Hamiltonian at first sight does not seem feasible because the absence of time-dependent forces implies energy conservation. However, as we will see below, once it is realized that dissipation arises from the coupling to other degrees of freedom, it is straightforward to model a damped quantum system in terms of a Hamiltonian.

A well known technique to describe a statistical dynamics governed by fluctuations is given by the method of generalized master equations and the methodology of generalized Langevin equations. This strategy is by now well developed for thermal equilibrium systems. Here the projector operator technique ${ }^{17-20,25}$ yields a clear-cut method to obtain the formal equations, either the (generally nonlinear) generalized quantum Langevin equation (QLE) or the generalized quantum master equation (QME) for the rate of change of the reduced density matrix.

Already for the case of relaxation towards a unique thermal equilibrium specified by a single temperature $T$, the equivalence between the two approaches is not very transparent. ${ }^{26} \mathrm{~A}$ crucial role is played by the fluctuational force which explicitly enters the equivalence, such as corresponding cumulant averages to an arbitrary high order. This fact is not appreciated generally, because one often restricts the discussion to the first two cumulants only, namely the average and its autocorrelation. It is a fact that little is known about the connection of the generalized master equation and the corresponding generalized Langevin equation in a nonlinear situation. ${ }^{26,27}$

A popular model for the dynamics of a dissipative quantum system subject to quantum Brownian noise is obtained by coupling the system of interest to a bath of harmonic oscillators. Accordingly, we write for the total Hamiltonian

$$
\begin{aligned}
H= & \frac{p^{2}}{2 M}+V(q, t) \\
& +\sum_{i=1}^{N}\left[\frac{p_{i}^{2}}{2 m_{i}}+\frac{m_{i}}{2} \omega_{i}^{2} x_{i}^{2}-q c_{i} x_{i}+q^{2} \frac{c_{i}^{2}}{2 m_{i} \omega_{i}^{2}}\right],
\end{aligned}
$$

where the first two terms describe the system as a particle of mass $M$ moving in a generally time-dependent potential $V(q, t)$. The sum contains the Hamiltonian for a set of $N$ harmonic oscillators which are bilinearly coupled with strength $c_{i}$ to the system. Finally, the last term, which depends only on the system coordinate, represents a potential renormalization term which is needed to ensure that $V(q, t)$ remains the bare potential. This Hamiltonian has been studied since the early 1960's for systems which are weakly coupled to the environmental degrees of freedom. ${ }^{9,18-20,28-31}$ Only after 1980, it was realized by Caldeira and Leggett ${ }^{32}$ that this model is also applicable to strongly damped systems and may be employed to describe, for example, dissipative tunnelling in solid state physics and chemical physics. ${ }^{31}$

One may convince oneself that the Hamiltonian (20) indeed models dissipation. Making use of the solution of the Heisenberg equations of motion for the external degrees of freedom $^{33}$ one derives a reduced system operator equation of motion, the so-called generalized quantum Langevin equation ${ }^{9}$

$$
M \ddot{q}(t)+M \int_{t_{0}}^{t} d s \gamma(t-s) \dot{q}(s)+\frac{d V(q, t)}{d q}=\xi(t)
$$

with the damping kernel

$$
\gamma(t)=\gamma(-t)=\frac{1}{M} \sum_{i=1}^{N} \frac{c_{i}^{2}}{m_{i} \omega_{i}^{2}} \cos \left(\omega_{i} t\right)
$$

and the quantum Brownian force operator

$$
\begin{aligned}
\xi(t)= & -M \gamma\left(t-t_{0}\right) q\left(t_{0}\right)+\sum_{i=1}^{N} c_{i}\left(x_{i}\left(t_{0}\right) \cos \left(\omega_{i}\left[t-t_{0}\right]\right)\right. \\
& \left.+\frac{p_{i}\left(t_{0}\right)}{m_{i} \omega_{i}} \sin \left(\omega_{i}\left[t-t_{0}\right]\right)\right) .
\end{aligned}
$$

The generalized quantum Langevin equation (21) appears first in a paper by Magalinski1 ${ }^{9}$ who started from (20) in the absence of the potential renormalization term.

The force operator (23) depends explicitly on the initial conditions at time $t_{0}$ of the bath position operators $x_{i}\left(t_{0}\right)$ and bath momenta $p_{i}\left(t_{0}\right)$. The initial preparation of the total system, which fixes the statistical properties of the bath operators and the system degrees of freeedom, turns the force $\xi(t)$ into a random operator. Note that this operator depends not only on the bath properties but as well on the initial system position $q\left(t_{0}\right)$. To fully specify the reduced dynamics it is thus of importance to specify the preparation procedure. This in turn then also fixes the statistical properties of the quantum Brownian noise. Clearly, in order to qualify as a stochastic force the random force $\xi(t)$ should not be biased; i.e. its average should be zero at all times. Moreover, this Brownian quantum noise should constitute a stationary process with time-homogeneous correlations.

Let us also introduce the auxiliary random force $\eta(t)$, defined by

$$
\eta(t)=\xi(t)+M \gamma\left(t-t_{0}\right) q\left(t_{0}\right)
$$

which only involves bath operators. In terms of this new random force the QLE (21) no longer assumes the form of an ordinary generalized Langevin equation: it now contains an inhomogeneous term $\gamma\left(t-t_{0}\right) q\left(t_{0}\right)$, the initial slip term. ${ }^{24,27}$ This term is often neglected in the so-called "Markovian limit" when the friction kernel assumes the Ohmic form $\gamma(t) \rightarrow 2 \gamma \delta(t)$. For a correlation-free preparation, the initial total density matrix is given by the product $\rho_{T}=\rho_{S}\left(t_{0}\right) \rho_{\text {bath }}$, where $\rho_{S}\left(t_{0}\right)$ is the initial system density matrix. The density matrix of the bath alone assumes canonical equilibrium, i.e., 


$$
\rho_{\text {bath }}=\frac{1}{\mathcal{N}} \exp \left(-\beta \sum_{i=1}^{N}\left[\frac{p_{i}^{2}}{2 m_{i}}+\frac{m_{i}}{2} \omega_{i}^{2} x_{i}^{2}\right]\right),
$$

with $\mathcal{N}$ denoting a normalization constant.

The statistical properties of the random force $\eta(t)$ then follow immediately: $\eta(t)$ is a stationary Gaussian operator noise obeying

$$
\begin{aligned}
\langle\eta(t)\rangle_{\rho_{\text {bath }}} & =0, \\
S_{\eta \eta}(t-s) & =\frac{1}{2}\langle\eta(t) \eta(s)+\eta(s) \eta(t)\rangle_{\rho_{\text {bath }}} \\
& =\frac{\hbar}{2} \sum_{i=1}^{N} \frac{c_{i}^{2}}{m_{i} \omega_{i}} \cos \left(\omega_{i}(t-s)\right) \operatorname{coth}\left(\frac{\hbar \omega_{i}}{2 k_{B} T}\right) .
\end{aligned}
$$

Being an operator-valued noise, its commutator does not vanish

$$
[\eta(t), \eta(s)]=-i \hbar \sum_{i=1}^{N} \frac{c_{i}^{2}}{m_{i} \omega_{i}} \sin \left(\omega_{i}(t-s)\right) .
$$

Setting for the initial position operator $q\left(t_{0}\right)=q_{0}$, the last expression in (27) is also valid for the noise correlation $S_{\xi \xi}(t)$ of the noise force $\xi(t)$ provided the average is now taken with respect to a bath density matrix which contains shifted oscillators. The initial preparation of the bath is then given by the new density matrix $\hat{\rho}_{\text {bath }}$;

$$
\hat{\rho}_{\text {bath }}=\frac{1}{\mathcal{N}} \exp \left\{-\beta \sum_{i}\left[\frac{p_{i}^{2}}{2 m_{i}}+\frac{m_{i} \omega_{i}^{2}}{2}\left(x_{i}-\frac{c_{i}}{m_{i} \omega_{i}^{2}} q_{0}\right)^{2}\right]\right\} .
$$

In some physical situations a microscopic model for the external degrees of freedom is available. ${ }^{31,34}$ Examples are the electromagnetic modes in a resonator acting as a reservoir or the dissipation arising from quasiparticle tunnelling through Josephson junctions. ${ }^{35}$ In the case of an electrical circuit containing a resistor one may use the classical equation of motion to obtain the damping kernel and model the environment accordingly. This approach has been used, e.g., to model Ohmic dissipation in Josephson junctions in order to study its influence on tunnelling processes,${ }^{36}$ and to describe the influence of an external impedance in the charge dynamics of ultrasmall tunnel junctions. ${ }^{37}$

This scheme of the QLE can also be extended to the nonequilibrium case with the system attached to two baths of different temperature. ${ }^{38} \mathrm{~A}$ most recent application addresses the problem of the thermal conductance through molecular wires that are coupled to leads of different temperature. Then the heat current assumes a form similar to the Landauer formula for electronic transport: The heat current is given in terms of a transmission factor times the difference of corresponding Bose functions. ${ }^{39}$

Furthermore, the QLE concept can also be extended to fermionic systems coupled to electron reservoirs and which, in addition, may be exposed to time-dependent driving. ${ }^{40}$ The corresponding Gaussian quantum noise is now composed of fermion annihilation operators.

\section{B. Consequences of time-reversal symmetry}

Let us now discuss some further properties of this QLE. If the potential $V(q, t)$ in (20) does not explicitly depend on time $t$, the dynamics of the full Hamiltonian (20) obeys time reversal symmetry. It is thus an immediate consequence that the reduced dynamics must be invariant under time reversal as well. This must hold true despite the fact that the QLE has been constructed to allow for a description of quantum dissipation. It is thus instructive to see how the validity under time reversal emerges from the contracted description in terms of the QLE in (21).

Given the time of preparation $t_{0}$, reversing the time amounts to substituting time $t$ by $t_{0}-\left(t-t_{0}\right)=2 t_{0}-t$. Using again the random force $\eta(t)$ we can recast the QLE dynamics after the time reversal into the form

$$
\begin{gathered}
M \ddot{q}\left(2 t_{0}-t\right)+M \int_{t_{0}}^{2 t_{0}-t} d s \gamma\left(2 t_{0}-t-s\right) \dot{q}(s)+\frac{d V(q)}{d q} \\
=\xi\left(2 t_{0}-t\right)=\eta\left(2 t_{0}-t\right)-\gamma\left(2 t_{0}-t-t_{0}\right) q\left(t_{0}\right) .
\end{gathered}
$$

Setting next $x(t)=q\left(2 t_{0}-t\right)$ and observing that $\dot{x}(t)$ $=-\dot{q}\left(2 t_{0}-t\right), \ddot{x}(t)=\ddot{q}\left(2 t_{0}-t\right)$, we find after the substitution of the integration time $u=2 t_{0}-s$ from (30) the result

$$
\begin{aligned}
M \ddot{x}(t) & +M \int_{t_{0}}^{t} d u \gamma(u-t) \dot{x}(u)+\frac{d V(q)}{d q} \\
= & \xi\left(2 t_{0}-t\right)=\eta\left(2 t_{0}-t\right)-\gamma\left(t_{0}-t\right) x\left(t_{0}\right) .
\end{aligned}
$$

Noting that the damping kernel is an even function of its argument, $\gamma(u-t)=\gamma(t-u)$, and that $x\left(t_{0}\right)=q\left(t_{0}\right)$, we find upon changing all signs of the initial momenta $p_{i}\left(t_{0}\right)$ $\rightarrow-p_{i}\left(t_{0}\right)$ for the noise forces the relations $\eta\left(2 t_{0}-t\right)=\eta(t)$ and $\xi\left(2 t_{0}-t\right)=\xi(t)$. We conclude that the time reversed motion $x(t)=q\left(2 t_{0}-t\right)$ indeed obeys again a QLE of the form (21). This even holds true in the Markovian limit where $\gamma(t-s)=2 \gamma \delta(t-s)$ as one can convince oneself by smearing out the delta function symmetrically. The QLE then reads for all times $t$

$$
M \ddot{q}(t)+\operatorname{sgn}\left(t-t_{0}\right) M \gamma \dot{q}(t)+\frac{d V(q)}{d q}=\xi(t),
$$

where $\operatorname{sgn}(x)$ denotes the sign of $x$.

The dissipation is reflected by the fact that for times $t$ $>t_{0}$ the reduced dynamics for $q(t)$ exhibits a damped (quantum-)behavior on a time scale given by the Poincaré recurrence time; ${ }^{41,42}$ the latter reaches essentially infinity for all practical purposes if only the bath consists of a sizable number of bath oscillator degrees of freedom. ${ }^{31,41}$

\section{Subtleties and pitfalls}

The use of the generally nonlinear QLE (21) is limited in practice for several reasons. Moreover, the application of the QLE bears some subtleties and pitfalls which must be observed when making approximations. Some important features are: The QLE (21) is an operator equation that acts in the full Hilbert space of system and bath. The coupling between system and environment also implies an entanglement upon time evolution even for the case of an initially factor- 
izing full density matrix. Together with the commutator property of quantum Brownian motion, see Eq. (28), we find that the reduced, dissipative dynamics of the position operator $q(t)$ and momentum operator $p(t)$ obey the Heisenberg uncertainty relation for all times.

This latter feature is crucial. For example, the nonMarkovian (colored) Gaussian quantum noise with realvalued correlation $S_{\xi \xi}(t)=S_{\xi \xi}(-t)$ cannot simply be substituted by a classical non-Markovian Gaussian noise force which identically obeys the correlation properties of (Gaussian) quantum noise $\xi(t)$. An approximation of this type clearly would not satisfy the commutator property for position and conjugate momentum of the system degrees of freedom.

The literature is full of various attempts wherein one approximates the quantum features by corresponding colored classical noise sources, e.g., see Refs. 43-45. Such schemes work at best near a quasiclassical limit, ${ }^{44,46}$ but even then care must be exercised. For example, for problems that exhibit an exponential sensitivity, as, e.g., the dissipative decay of a metastable state discussed in the next section, such an approach gives no exact agreement with the quantum dissipative theory. ${ }^{31,34}$ It is only in the classical high temperature limit, where the commutator structure of quantum mechanics no longer influences the result. Perfect agreement is only achieved in the classical limit.

The study of quantum friction in a nonlinear quantum system by means of the QLE (21) is plagued by the fact that the nonlinearity forbids an explicit solution. This solution, however, is needed to obtain the statistical properties such as mean values and correlation functions. This (unknown) nonlinear response function also determines the derivation of the rate of change of the reduced density operator, i.e. the QME, and its solution for the open quantum system.

The very fact that the QLE acts in full Hilbert space of system and environment also needs to be distinguished from the classical case of a generalized Langevin equation. There, the stochastic dynamics acts solely on the state space of the system dynamics with the (classical) noise properties specified $a$ priori. $^{47}$

The quantum noise correlations can, despite the explicit microscopic expression given in (27), be expressed solely by the macroscopic friction kernel $\gamma(t)$. This result follows upon noting that the Laplace transform $\hat{\gamma}(z)$ of the macroscopic friction assumes with $\operatorname{Re} z>0$ the form

$$
\hat{\gamma}(z)=\frac{1}{2 M} \sum_{i=1}^{N} \frac{c_{i}^{2}}{m_{i} \omega_{i}^{2}}\left[\frac{1}{z-i \omega_{i}}+\frac{1}{z+i \omega_{i}}\right] .
$$

With help of the well known relation $1 /\left(x+i 0^{+}\right)=P(1 / x)$ $-i \pi \delta(x)$ we find that

$$
\begin{aligned}
\operatorname{Re} \hat{\gamma}(z & \left.=-i \omega+0^{+}\right) \\
& =\frac{\pi}{2 M} \sum_{i=1}^{N} \frac{c_{i}^{2}}{m_{i} \omega_{i}^{2}}\left[\delta\left(\omega-\omega_{i}\right)+\delta\left(\omega+\omega_{i}\right)\right] .
\end{aligned}
$$

By means of (27) we then find the useful relation

$$
\begin{aligned}
S_{\xi \xi}(t) & =S_{\eta \eta}(t) \\
& =\frac{M}{\pi} \int_{0}^{\infty} d \omega \operatorname{Re} \hat{\gamma}\left(-i \omega+0^{+}\right) \hbar \omega \operatorname{coth}\left(\frac{\hbar \omega}{2 k_{B} T}\right) \cos (\omega t) .
\end{aligned}
$$

In the classical limit this relation reduces, independent of the preparation of the bath with $\rho$ or $\hat{\rho}$, to the non-Markovian Einstein relation $S_{\xi \xi}(t)=M k_{B} T \gamma(t)$. The relation (35) is by no means obvious: It implies that a modelling of quantum dissipation is possible in terms of macroscopic quantities such as the friction kernel $\gamma(t)$ and the temperature $T$. For other coupling schemes between system and bath we generally can no longer express the correlation of quantum noise exclusively in terms of macroscopic transport coefficients. As an example we mention the coupling of the system to a bath of two-level systems (spin bath) rather than to a bath of harmonic oscillators. $^{48}$

Note, also the following differences to the classical situation of a generalized Langevin equation: The quantum noise $\xi(t)$ is correlated with the initial position operator $q\left(t_{0}\right){ }^{49}$ This feature that $\left\langle q\left(t_{0}\right) \xi(t)\right\rangle_{\hat{\rho}} \neq 0$ follows from the explicit form of the quantum noise $\xi(t)$. The correlation function vanishes only in the classical limit. Note also that the expectation value of the system-bath interaction is finite at zero temperature. These features reflect the fact that at absolute zero temperature the coupling induces a nonvanishing decoherence via the zero-point fluctuations.

Moreover, the initial slip term $\gamma\left(t-t_{0}\right) q\left(t_{0}\right)$ appears also in the absence of the potential renormalization in the Hamiltonian (20). With this initial value contribution being absorbed into the quantum fluctuation $\xi(t)$, these become stationary fluctuations with respect to the initial density operator of the bath $\hat{\rho}_{\text {bath }}$ given by (29). Note, however, that with respect to an average over the bare, nonshifted bath density operator $\rho_{\text {bath }}$, the quantum fluctuations $\xi(t)$ would become nonstationary.

It is also worthwhile to point out here that this initial value term in the QLE should not be confused with the initial value term that enters the corresponding QME. ${ }^{17,18}$ In the case of a classical reduced dynamics it is always possible - by use of a corresponding projection operator-to formally eliminate this initial, inhomogeneous contribution in the generalized master equation. ${ }^{47,50}$ This in turn renders the time evolution of the reduced probability a truly linear dynamics. This property no longer holds for the reduced quantum dynamics. ${ }^{51}$ For a non-factorizing initial preparation of system and bath this initial value contribution in the QME generally is finite and presents a true nonlinearity for the time evolution law of the open quantum dynamics!

There exist even further subtleties which are worthwhile to point out. The friction enters formally the QLE just in the same way as in the classical generalized Langevin equation. In particular, a time-dependent potential $V(q, t)$ leaves this friction kernel invariant in the QLE. In contrast to the classical Markovian case, however, where the friction enters the corresponding Fokker-Planck dynamics independent of the 
time scale of driving, this is no longer valid for the generalized quantum master equation dynamics of the corresponding reduced density matrix. ${ }^{52,53}$

The solution of the QLE involves the explicit timedependence of both the friction and the potential forces. These in turn determine the statistical properties of the density matrix. As a consequence, the friction force enters the QME in a rather complex manner. This can already be verified explicitly for a parametric dissipative oscillator dynamics, where the time-dependent driving enters the diffusive kinetic evolution law of the reduced density operator or its equivalent Wigner transform. ${ }^{52,53}$

For the bilinear system-bath interaction with the bath composed of harmonic oscillators it was possible to integrate out the degrees of freedom of the bath explicitly. Does this hold as well for other interactions? The elimination of the bath degrees of freedom is still possible for a nonlinear coupling to a bath of harmonic oscillators if the system part of the coupling is replaced by a nonlinear operator-valued function of either the momentum or position degree of freedom of the system as long as the bath degrees of freedom appear linearly. The resulting friction kernel then appears as a nonlinear friction but the influence of the bath degrees of freedom still is obtained in exact form. ${ }^{27}$

Yet another situation for which one can derive an exact QLE is when a nonlinear system, such as a spin degree of freedom, interacts with a collection of quantum (Bose) oscillators in such a way that the interaction Hamiltonian commutes with the system Hamiltonian, thus constituting a quantum nondemolition interaction. This case corresponds to pure dephasing and was addressed by Łuczka for the problem of a spin in contact with a thermal heat bath. ${ }^{54}$ It has since been rederived many times, see, e.g., Ref. 55.

We end this subsection by mentioning also the coupling of a system to a bath of independent fermions with infinitely many excitation energies. A suitable transformation then allows to map the dissipation onto a bosonic environment with an appropriate coupling strength. ${ }^{31,40,56}$

\section{PATH INTEGRALS AND EFFECTIVE ACTION}

\section{A. Nonlocal effective action}

A most effective approach to describe dissipation is based on the path integral formulation of quantum mechanics. ${ }^{57}$ In the path integral formulation of quantum mechanics the propagator is expressed as

$$
\left\langle q_{f}\left|\exp \left(-\frac{i}{\hbar} H t\right)\right| q_{i}\right\rangle=\int_{q(0)=q_{i}}^{q(t)=q_{f}} \mathcal{D} q \exp \left(\frac{i}{\hbar} S[q]\right),
$$

where the integral runs over all possible paths starting at $q_{i}$ and ending after time $t$ at $q_{f}$. The paths are weighted with a phase factor which contains the classical action $S[q]$.

For the description of quantum dissipative systems it is important to realize the analogy between the propagator and the equilibrium density matrix. The latter is obtained by replacing $t$ by $-i \hbar \beta$. We thus obtain from (36) the path integral representation of the equilibrium density matrix

$$
\rho_{\beta}\left(q, q^{\prime}\right)=\frac{1}{Z_{\beta}} \int_{q(0)=q^{\prime}}^{q(\hbar \beta)=q} \mathcal{D} q \exp \left(-\frac{1}{\hbar} S^{E}[q]\right),
$$

where $Z_{\beta}$ is the partition function. This integral is called imaginary-time path integral in contrast to the real-time path integral (36). Note that in (37) the action $S[q]$ has been replaced by the so-called Euclidean action $S^{E}[q]$ which is obtained by changing the sign of the potential term as a consequence of the transition to imaginary times. In imaginary time we therefore have to consider the motion in the inverted potential.

The connection between classical and quantum mechanics becomes particularly apparent in the path integral formulation. The dominant contribution to the integrals in (36) and (37) arise from the stationary points of the action, i.e., the classical paths. Quantum effects have their origin in fluctuations around the classical paths. Therefore, it is useful to decompose a general path into the classical path and a fluctuation around it. Expanding the action in powers of the fluctuations the second order term yields the leading quantum corrections. Higher order terms are often neglected within a semiclassical approximation which becomes exact for linear systems.

In the previous section we have derived an effective equation of motion for the system variable by eliminating the external degrees of freedom. The same procedure may of course also be carried out within the path integral formalism. $6,31,34,58$ The influence of the environment is then contained in an effective action which has to be added to the action of the system and which in imaginary time is given by $^{6,31,34}$

$$
S_{\mathrm{eff}}[q]=-\frac{1}{4} \int_{0}^{\hbar \beta} d \tau \int_{0}^{\hbar \beta} d \sigma k(\tau-\sigma)[q(\tau)-q(\sigma)]^{2},
$$

where

$$
k(\tau)=\frac{M}{\hbar \beta} \sum_{n=-\infty}^{+\infty}\left|\nu_{n}\right| \hat{\gamma}\left(\left|\nu_{n}\right|\right) \exp \left(i \nu_{n} \tau\right)
$$

and $\hat{\gamma}(z)$ denotes the Laplace transform of the damping kernel $\gamma(t)$. The effective action (38) is clearly nonlocal and can thus not be expressed in terms of a potential. If the potential renormalization term in the Hamiltonian (20) would be absent, there would have been a local contribution in (38). The selfinteraction of the paths induced by (38) via the kernel (39) decays for Ohmic damping only algebraically as $\tau^{-2}$ and therefore represents a long range interaction.

\section{B. Application: The dissipative decay of a metastable state}

A local potential minimum may be metastable due to the environmental coupling and quantum effects. Correspondingly, there are two escape mechanisms: thermal activation which dominates at high temperatures and quantum tunnelling which becomes important at low temperatures. To be definite, we consider the cubic potential 


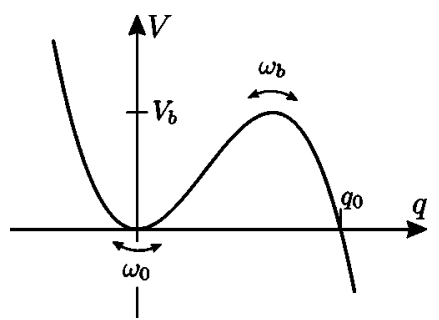

FIG. 3. Cubic potential as defined in Eq. (40).

$$
V(q)=\frac{M}{2} \omega_{0}^{2} q^{2}\left(1-\frac{q}{q_{0}}\right)
$$

which is depicted in Fig. 3. The barrier height is given by $V_{b}=(2 / 27) M \omega_{0}^{2} q_{0}^{2}$ and, in this special case, the barrier angular frequency $\omega_{b}$ equals the well angular frequency $\omega_{0}$.

In Fig. 4, the decay rate is shown in an Arrhenius plot. At the so-called crossover temperature $T_{0}$, see Eq. (42) below, there is a rather distinct transition between the thermal regime on the left-hand side and the quantum regime on the right-hand side. ${ }^{59}$ Furthermore, we observe that the thermal regime is larger for stronger damping, i.e., the system becomes more classical.

While a real time approach to dissipative decay is feasible, ${ }^{61-64}$ a simpler alternative is provided by an imaginary time calculation where the partition function $Z_{\beta}$ is considered. Since the potential (40) is not bounded from below, it is no surprise that strictly speaking $Z_{\beta}$ does not exist. From the path integral point of view there exists an unstable fluctuation mode around the barrier which leads to a saddle point in function space. One can circumvent this difficulty by performing the integration in the direction of steepest descent. The partition function and as a consequence also the free energy then acquire an imaginary part which may be related to the decay rate. ${ }^{31,32}$ For details of this relation we refer the reader to the discussion in Ref. 65 .

The transition between thermal and quantum regime can be well understood within the path integral picture by considering the possible classical paths of duration $\hbar \beta$ in the inverted cubic potential. For high temperatures or short imaginary times $\hbar \beta$ the only classical solutions are the con-

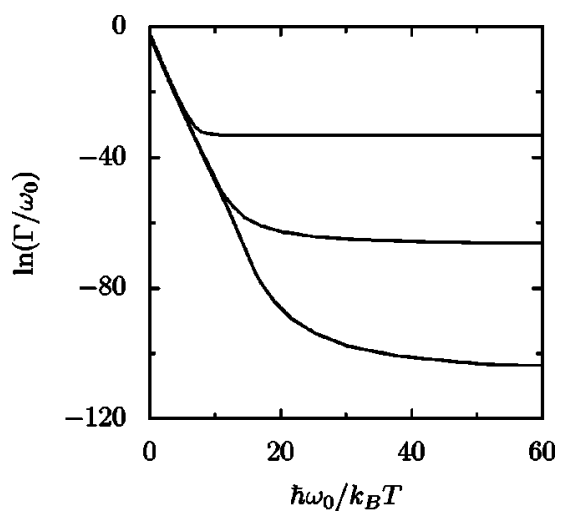

FIG. 4. Arrhenius plot for the decay rate of a metastable state. The damping strength varies from the upper to the lower curve as $\gamma / 2 \omega_{0}=0,0.5$, and 1 (data taken from Ref. 60). stant solutions $q=0$ in the well and $q_{b}=2 q_{0} / 3$ at the barrier. Below a temperature given by the positive solution of

$$
\nu_{1}^{2}+\left|\nu_{1}\right| \hat{\gamma}\left(\left|\nu_{1}\right|\right)-\omega_{b}^{2}=0
$$

a second fluctuation mode becomes unstable, thereby indicating a new classical solution which performs an oscillation around the barrier. ${ }^{66}$ This new solution is associated with quantum tunnelling. Therefore, (41) defines the crossover temperature which for Ohmic damping is given by ${ }^{59}$

$$
T_{0}=\frac{\hbar}{2 \pi k_{B}}\left[\left(\frac{\gamma^{2}}{4}+\omega_{b}^{2}\right)^{1 / 2}-\frac{\gamma}{2}\right] .
$$

As discussed above, stronger damping leads to a lower crossover temperature and smaller quantum regime. It thus makes the system more classical. A distinct feature of the dissipative quantum decay in the low temperature regime is its algebraic enhancement of the decay rate with temperature. ${ }^{31}$ For the case of an Ohmic environment with a constant friction behavior at low frequencies one finds a universal $T^{2}$-enhancement of both, the prefactor and the effective action, with the latter dominating the exponential rate enhancement. ${ }^{31,67}$

\section{SUNDRY REMARKS AND CONCLUSIONS}

With this work we elucidated the topic of quantum Brownian noise which drives the dynamics of open dissipative quantum systems. We have emphasized the strong implications that thermal equilibrium and time-reversal symmetry (leading to detailed balance symmetry) imposes on the reduced system dynamics. We also pointed out the advantageous use of the path integral scheme for the case of nonlinearity and strong friction.

This method seemingly is superior to any perturbative scheme that treats the system-bath coupling to low orders only, such as the weak coupling master equation methodology. ${ }^{17-20}$ There are recent developments in the strong friction regime, where an alternative description in terms of a quantum Smoluchowski equation is promising, ${ }^{68}$ see also the contribution by Grabert, Ankerhold, and Pechukas in this Focus Issue.

A consequent use of the so-called rotating-wave approximations also may entail some danger. It safely can be applied only in the weak coupling regime for resonant situations. We remark that the use of the rotating-wave approximation implies a violation of the Ehrenfest theorem in the order of $\gamma^{2},{ }^{13,69}$ which is clearly small only in the weak coupling regime, i.e., for $\gamma \ll \omega_{0}$, with $\omega_{0}^{-1}$ denoting some typical time scale of the system dynamics. The same remarks apply to the failure of the quantum regression theorem: ${ }^{13,70,71}$ Again, the effect might be small for (i) very weak damping, (ii) not too low temperatures obeying $k_{B} T \gg \hbar \gamma$, and (iii) not too short evolution times.

The generalized quantum Langevin equation discussed in Sec. IV is formally exact for nonlinear quantum systems. Its practical use is typically restricted, however, to linear systems for which the response can be evaluated in closed form. This holds true even for time-dependent linear systems for which the response is still linear although the evaluation 
involves the use of numerical Floquet theory. ${ }^{52}$ The lack of knowledge of this generally nonlinear response function also plagues the evaluation of the corresponding generalized master equations.

This problem of obtaining the generalized master equation from the nonlinear generalized Langevin equation is not solved either for the classical problem with colored noise. ${ }^{27}$ It is also this very problem that limits the practical use of the various variants of recently derived stochastic Schrödinger approaches. $^{12}$

Likewise, the use of nonlinear, but nonstochastic Schrödinger equations of the type discussed and surveyed in Refs. 72-74 can clearly not describe the time evolution of a quantum mechanical mixture, nor do these nonlinear deterministic approaches obey, in general, the Heisenberg uncertainty relation. ${ }^{73,74}$

There have been repeated attempts since the early days of quantum mechanics to explain quantum phenomena in terms of Einstein's theory of classical diffusion. Early efforts in this direction were those of Fürth, ${ }^{75}$ Fényes, ${ }^{76}$ Weizel, ${ }^{77}$ and Favella. ${ }^{78}$ This credo has been popularized later by Nelson ${ }^{10,79}$ under the label of "Stochastic Mechanics." It can convincingly be demonstrated, however, that a quantum dynamics is quite distinct from a classical Markovian - or even non-Markovian-stochastic dynamics. ${ }^{11,80}$ This holds even more so, if one attempts to incorporate the quantum dissipation for an open system.

These sundry remarks thus give clear evidence that the topic of quantum Brownian motion-although 100 years have passed since Einstein's cornerstone contribution — cannot be considered as "solved." For example, little is presently known also for the description and the role of quantum noise acting in steady state, far from equilibrium situations, i.e., when several baths of different nature and/or different temperature are coupled to the nonlinear system of interest.

The latter case is also of salient importance for the description of the quantum dynamics of so-called quantum Brownian motors. ${ }^{81}$ In those applications quantum Brownian noise is utilized in combination with nonequilibrium (classical or quantum) fluctuations to perform exploitable work against external bias forces. In summary, the field of quantum Brownian motion is very much alive and lots of challenges still need to be addressed and mastered.

\section{ACKNOWLEDGMENTS}

P.H. gratefully acknowledges financial support by the DAAD-KBN (German-Polish project Stochastic Complexity), the Foundation for Polish Science (Fundacja na Rzecz Nauki Polskiej), the Deutsche Forschungsgemeinschaft via Grant No. HA 1517/13-4 and the collaborative research centers SFB 486 and SFB 631.

${ }^{1}$ A. Einstein, "Über die von der molekularkinetischen Theorie der Wärme geforderte Bewegung von in ruhenden Flüssigkeiten suspendierten Teilchen," Ann. Phys. 17, 549-560 (1905).

${ }^{2}$ H. Nyquist, "Thermal agitation of electric charge in conductors," Phys. Rev. 32, 110-113 (1928).

${ }^{3}$ J. B. Johnson, "Thermal agitation of electricity in conductors," Phys. Rev. 32, 97-109 (1928).
${ }^{4}$ It is worthwhile to recall that the validity of the equipartition theorem is restricted to classical statistical mechanics.

${ }^{5}$ H. B. Callen and T. A. Welton, "Irreversibility and generalized noise," Phys. Rev. 83, 34-40 (1951).

${ }^{6}$ H. Grabert, P. Schramm, and G.-L. Ingold, "Quantum Brownian motion: The functional integral approach,” Phys. Rep. 168, 115-207 (1988).

${ }^{7}$ T. Dittrich, P. Hänggi. G.-L. Ingold, B. Kramer, G. Schön, and W. Zwerger, Quantum Transport and Dissipation (Wiley-VCH, Weinheim, 1998).

${ }^{8}$ M. Grifoni and P. Hänggi, "Driven quantum tunneling," Phys. Rep. 304, 229-358 (1998).

${ }^{9} \mathrm{~V}$. B. Magalinski1, "Dynamical model in the theory of the Brownian motion," Sov. Phys. JETP 9, 1381-1382 (1959) [J. Exp. Theor. Phys. 36, 1942-1944 (1959)]; R. Benguria and M. Kac, "Quantum Langevin equation," Phys. Rev. Lett. 46, 1-4 (1981); G. W. Ford and M. Kac, "On the quantum Langevin equation,” J. Stat. Phys. 46, 803-810 (1987); G. W. Ford, J. T. Lewis, and R. F. O'Connell, "Quantum Langevin equation," Phys. Rev. A 37, 4419-4428 (1988).

${ }^{10}$ E. Nelson, "Derivation of the Schrödinger equation from Newtonian mechanics," Phys. Rev. 150, 1079-1085 (1966).

${ }^{11} \mathrm{H}$. Grabert, P. Hänggi, and P. Talkner, "Is quantum mechanics equivalent to a classical stochastic process?" Phys. Rev. A 19, 2440-2445 (1979).

${ }^{12} \mathrm{M}$. Roncadelli, "Connection between Langevin quantization and classical mechanics," Europhys. Lett. 28, 379-384 (1994); H. Kleinert and S. V. Shabanov, "Quantum Langevin equation from forward-backward path integral," Phys. Lett. A 200, 224-232 (1995); L. Diósi and W. T. Strunz, "The non-Markovian stochastic Schrödinger equation for open systems," ibid. 235, 569-573 (1997); W. T. Strunz, L. Diósi, and N. Gisin, "Open system dynamics with non-Markovian quantum trajectories," Phys. Rev. Lett. 82, 1801-1805 (1999); H. P. Breuer, B. Kappler, and F. Petruccione, "Stochastic wave function method for non-Markovian quantum master equations," Phys. Rev. A 59, 1633-1643 (1999); J. T. Stockburger and H. Grabert, "Non-Markovian quantum state diffusion," Chem. Phys. 268, 249-256 (2001); J. Shao, "Decoupling quantum dissipation interaction via stochastic fields," J. Chem. Phys. 120, 5053-5056 (2004).

${ }^{13} \mathrm{P}$. Talkner, "The failure of the quantum regression hypothesis," Ann. Phys. (N.Y.) 167, 390-436 (1986); "Untersuchungen irreversibler Prozesse in quantenmechanischen Systemen," Ph.D. thesis, Universität Stuttgart, 1979.

${ }^{14}$ M. Planck, "Eine neue Strahlungshypothese," Verh. Dtsch. Phys. Ges. 13, 138-148 (1911).

${ }^{15} \mathrm{H}$. Grabert, U. Weiss, and P. Talkner, "Quantum theory of the damped harmonic oscillator," Z. Phys. B: Condens. Matter 55, 87-94 (1984).

${ }^{16}$ P. S. Riseborough, P. Hänggi, and U. Weiss, "Exact results for a damped quantum-mechanical harmonic oscillator," Phys. Rev. A 31, 471-478 (1985).

${ }^{17}$ H. Grabert, Projection Operator Techniques in Nonequilibrium Statistical Mechanics, Springer Tracts in Modern Physics (Springer, Berlin, 1982), Vol. 95, pp. 1-164.

${ }^{18}$ F. Haake, Statistical Treatment of Open Systems by Generalized Master Equations, Springer Tracts in Modern Physics (Springer, Berlin, 1973), Vol. 66, pp. 98-168.

${ }^{19}$ H. Spohn, "Kinetic equations from Hamiltonian dynamics: Markovian limits,” Rev. Mod. Phys. 52, 569-615 (1980).

${ }^{20} \mathrm{R}$. Alicki, "General theory and applications to unstable particles," in Quantum Dynamical Semigroups and Applications, Lect. Notes Phys. (Springer, Berlin, 1987), Vol. 286, Chaps. II and III.

${ }^{21}$ G.-L. Ingold and H. Grabert, "Sluggish decay of preparation effects in low temperature quantum systems," Lect. Notes Math. 1442, 219-230 (1990).

${ }^{22}$ R. Jung, G.-L. Ingold, and H. Grabert, "Long-time tails in quantum Brownian motion," Phys. Rev. A 32, 2510-2512 (1985).

${ }^{23}$ A. Hanke and W. Zwerger, "Density of states of a damped quantum oscillator," Phys. Rev. E 52, 6875-6878 (1995).

${ }^{24}$ G.-L. Ingold, "Path integrals and their application to dissipative quantum systems," Lect. Notes Phys. 611, 1-53 (2002).

${ }^{25} \mathrm{H}$. Mori, "Transport, collective motion and Brownian motion," Prog. Theor. Phys. 33, 423-455 (1965); K. Kawasaki, "Simple derivations of generalized linear and nonlinear Langevin equations," J. Phys. A 6, 12891295 (1973); S. Nordholm and R. Zwanzig, "Systematic derivation of exact generalized Brownian-motion theory," J. Stat. Phys. 13, 347-371 (1975).

${ }^{26} \mathrm{H}$. Grabert, P. Hänggi, and P. Talkner, "Microdynamics and nonlinear stochastic processes of gross variables," J. Stat. Phys. 22, 537-552 (1980).

${ }^{27}$ P. Hänggi, "Generalized Langevin equations: A useful tool for the per- 
plexed modeller of nonequilibrium fluctuations?" Lect. Notes Phys. 484, 15-22 (1997).

${ }^{28}$ I. R. Senitzky, "Dissipation in quantum mechanics. The harmonic oscillator," Phys. Rev. 119, 670-679 (1960).

${ }^{29}$ P. Ullersma, "An exactly solvable model for Brownian motion," Physica (Utrecht) 32, 27-55 (1966); 32, 56-73 (1966); 32, 74-89 (1966); 32, 90-96 (1966)

${ }^{30}$ R. Zwanzig, "Nonlinear generalized Langevin equations," J. Stat. Phys. 9, 215-220 (1973).

${ }^{31}$ P. Hänggi, P. Talkner, and M. Borkovec, "Reaction-rate theory: Fifty years after Kramers," Rev. Mod. Phys. 62, 251-341 (1990).

${ }^{32}$ A. O. Caldeira and A. J. Leggett, "Quantum tunnelling in a dissipative system," Ann. Phys. (N.Y.) 149, 374-456 (1983); 153, 445 (1984)

${ }^{33}$ For the explicit details of this calculation, see Refs. 24 and 27.

${ }^{34}$ U. Weiss, Quantum Dissipative Systems, 2nd ed. (World Scientific, Singapore, 1999).

${ }^{35}$ U. Eckern, G. Schön, and V. Ambegaokar, "Quantum dynamics of a superconducting tunnel junction,” Phys. Rev. B 30, 6419-6431 (1984).

${ }^{36}$ G. Schön and A. D. Zaikin, "Quantum coherent effects, phase transitions, and the dissipative dynamics of ultra small tunnel junctions," Phys. Rep. 198, 237-413 (1990).

${ }^{37}$ G.-L. Ingold and Yu. V. Nazarov, "Charge tunneling rates in ultrasmall junctions," in Single Charge Tunneling, NATO ASI Series B, edited by H. Grabert and M. H. Devoret (Plenum, New York, 1992), Vol. 294, pp. 21-107.

${ }^{38}$ U. Zürcher and P. Talkner, "Quantum-mechanical harmonic chain attached to heat baths II. Nonequilibrium properties," Phys. Rev. A 42, 3278-3290 (1990).

${ }^{39}$ D. Dvira, A. Nitzan, and P. Hänggi, "Thermal conductance through molecular wires," J. Chem. Phys. 119, 6840-6855 (2003).

${ }^{40}$ S. Camalet, J. Lehmann, S. Kohler, and P. Hänggi, "Current noise in ac-driven nanoscale conductors," Phys. Rev. Lett. 90, 210602 (2003); S. Camalet, S. Kohler, and P. Hänggi, "Shot-noise control in ac-driven nanoscale conductors," Phys. Rev. B 70, 155326 (2004).

${ }^{41}$ P. C. Hemmer, L. C. Maximon, and H. Wergeland, "Recurrence time of a dynamical system," Phys. Rev. 111, 689-694 (1958).

${ }^{42}$ P. Mazur and E. Montroll, "Poincaré cycles, ergodicity, and irreversibility in assemblies of coupled harmonic oscillators," J. Math. Phys. 1, 70-84 (1960).

${ }^{43}$ S. A. Adelman, "Quantum generalized Langevin equation approach to gas/solid collisions," Chem. Phys. Lett. 40, 495-499 (1976).

${ }^{44}$ A. Schmid, "On a quasiclassical Langevin equation," J. Low Temp. Phys. 49, 609-626 (1982).

${ }^{45}$ S. K. Banik, B. C. Bag, and D. S. Ray, "Generalized quantum FokkerPlanck, diffusion and Smoluchowski equations with true probability distribution functions," Phys. Rev. E 65, 051106 (2002); D. Banerjee, B. C. Bag, S. K. Banik, and D. S. Ray, "Solution of quantum Langevin equation: Approximations, theoretical and numerical aspects," J. Chem. Phys. 120, 8960-8972 (2004).

${ }^{46}$ U. Eckern, W. Lehr, A. Menzel-Dorwarth, F. Pelzer, and A. Schmid, "The quasiclassical Langevin equation and its application to the decay of a metastable state and to quantum fluctuations," J. Stat. Phys. 59, 885-934 (1990).

${ }^{47}$ P. Hänggi and H. Thomas, "Stochastic processes: Time evolution, symmetries and linear response," Phys. Rep. 88, 207-319 (1982).

${ }^{48}$ J. Shao and P. Hänggi, "Decoherent dynamics of a two-level system coupled to a sea of spins," Phys. Rev. Lett. 81, 5710-5713 (1998).

${ }^{49}$ P. Schramm, R. Jung, and H. Grabert, "A closer look at the quantum Langevin equation: Fokker-Planck equation and quasiprobabilities," Phys. Lett. 107A, 385-389 (1985).

${ }^{50}$ H. Grabert, P. Talkner, and P. Hänggi, "Microdynamics and time-evolution of macroscopic non-Markovian systems," Z. Phys. B 26, 389-395 (1977); H. Grabert, P. Talkner, P. Hänggi, and H. Thomas, "Microdynamics and time-evolution of macroscopic non-Markovian systems II," ibid. 29, 273280 (1978)

${ }^{51}$ K. M. F. Romero, P. Talkner, and P. Hänggi, "Is the dynamics of open quantum systems always linear?” Phys. Rev. A 69, 052109 (2004).

${ }^{52}$ C. Zerbe and P. Hänggi, "Brownian parametric quantum oscillator with dissipation," Phys. Rev. E 52, 1533-1543 (1995).

${ }^{53}$ S. Kohler, T. Dittrich, and P. Hänggi, "Floquet-Markovian description of parametrically driven, dissipative harmonic oscillator," Phys. Rev. E 55, 300-313 (1997).

${ }^{54}$ J. Łuczka, "Spin in contact with thermostat: Exact reduced dynamics," Physica A 167, 919-934 (1990).

${ }^{55}$ N. G. van Kampen, "A soluble model for quantum mechanical dissipa- tion,” J. Stat. Phys. 78, 299-310 (1995).

${ }^{56}$ L.-D. Chang and S. Chakravarty, "Dissipative dynamics of a two-state system coupled to a heat bath," Phys. Rev. B 31, 154-164 (1985); F. Sols and F. Guinea, "Bulk and surface diffusion of heavy particles in metals: A path-integral approach," ibid. 36, 7775-7785 (1987); P. Hedegård and A. O. Caldeira, "Quantum dynamics of a particle in a fermionic environment," Phys. Scr. 35, 609-622 (1987).

${ }^{57}$ R. P. Feynman, "Space-time approach to nonrelativistic quantum mechanics," Rev. Mod. Phys. 20, 367-387 (1948); R. P. Feynman and A. R. Hibbs, Quantum Mechanics and Path Integrals (McGraw-Hill, New York, 1965).

${ }^{58}$ R. P. Feynman and F. L. Vernon, "The theory of a general quantum system interacting with a linear dissipative system," Ann. Phys. (N.Y.) 24, 118173 (1963).

${ }^{59}$ P. Hanggi, H. Grabert, G.-L. Ingold, and U. Weiss, "Quantum theory of activated events in presence of long-time memory," Phys. Rev. Lett. 55, 761-764 (1985).

${ }^{60}$ H. Grabert, P. Olschowski, and U. Weiss, "Quantum decay rates for dissipative systems at finite temperatures," Phys. Rev. B 36, 1931-1951 (1987).

${ }^{61}$ G.-L. Ingold, "Anwendung von Funktionalintegralen auf Transport- und Relaxationsphänomene in dissipativen Quantensystemen," Ph.D. thesis, Universität Stuttgart, 1988.

${ }^{62}$ H. Hofmann und G.-L. Ingold, "Dissipative transport across a parabolic barrier," Phys. Lett. B 264, 253-258 (1991).

${ }^{63}$ J. Ankerhold, H. Grabert, and G.-L. Ingold, "Dissipative quantum systems with potential barrier. General theory and parabolic barrier," Phys. Rev. E 51, 4267-4281 (1995); J. Ankerhold and H. Grabert, "Dissipative quantum systems with a potential barrier. II. Dynamics near the barrier top," ibid. 52, 4704-4723 (1995); Dissipative quantum systems with a potential barrier. III. Steady state nonequilibrium flux and reaction rate," ibid. $\mathbf{5 5}$, 1355-1374 (1997).

${ }^{64}$ J. Ankerhold and H. Grabert, "Quantum tunneling and the semiclassical real time dynamics of the density matrix," Europhys. Lett. 47, 285-291 (1999).

${ }^{65} \mathrm{P}$. Hänggi and W. Hontscha, "Periodic orbit approach to the quantumKramers-rate," Ber. Bunsenges. Phys. Chem. 95, 379-385 (1991); "Unified approach to the quantum-Kramers reaction rate," J. Chem. Phys. 88, 4094-4095 (1988).

${ }^{66}$ P. S. Riseborough, P. Hänggi, and E. Freidkin, "Quantum tunneling in dissipative media: Intermediate-coupling-strength results," Phys. Rev. A 32, 489-499 (1985)

${ }^{67}$ H. Grabert, U. Weiss, and P. Hanggi, "Quantum tunneling in dissipative systems at finite temperatures," Phys. Rev. Lett. 52, 2193-2196 (1984).

${ }^{68}$ J. Ankerhold, P. Pechukas, and H. Grabert, "Strong friction limit in quantum mechanics: The quantum Smoluchowski equation," Phys. Rev. Lett. 87, 086802 (2001); J. Ankerhold, "Quantum decay rates for driven barrier potentials in the strong friction limit," Phys. Rev. E 64, 060102 (2001); L. Machura, M. Kostur, P. Hänggi, P. Talkner, and J. Łuczka, "Consistent description of quantum Brownian motors operating at strong friction," ibid. 70, 031107 (2004)

${ }^{69}$ G. W. Ford and R. F. O'Connell, "Inconsistency of the rotating wave approximation with the Ehrenfest theorem," Phys. Lett. A 215, 245-246 (1996).

${ }^{70}$ H. Grabert, "Non-linear relaxation and fluctuations of damped quantum systems," Z. Phys. B: Condens. Matter 42, 161-172 (1982).

${ }^{71}$ G. W. Ford and R. F. O'Connell, “There is no quantum regression theorem," Phys. Rev. Lett. 77, 798-801 (1996).

${ }^{72}$ M. D. Kostin, "Friction and dissipative phenomena in quantum mechanics," J. Stat. Phys. 12, 145 (1975).

${ }^{73}$ J. Messer, "Friction in quantum mechanics," Acta Phys. Austriaca 50, 75-91 (1979), and references therein.

${ }^{74}$ H. Dekker, "Classical and quantum mechanics of the damped harmonic oscillator,” Phys. Rep. 80, 1-112 (1981).

${ }^{75}$ R. Fürth, "Über einige Beziehungen zwischen klassischer Statistik und Quantenmechanik," Z. Phys. 81, 143-162 (1933).

${ }^{76}$ I. Fényes, "Eine wahrscheinlichkeitstheoretische Begründung und Interpretation der Quantenmechanik," Z. Phys. 132, 81-106 (1952).

${ }^{77} \mathrm{~W}$. Weizel, “Ableitung der Quantentheorie aus einem klassischen, kausal determinierten Modell," Z. Phys. 134, 264-285 (1953); "Ableitung der Quantentheorie aus einem klassischen Modell. II,” ibid. 135, 270-273 (1953).

${ }^{78}$ L. F. Favella, "Brownian motions and quantum mechanics," Ann. Inst. Henri Poincare, Sect. A 7, 77-94 (1967).

${ }^{79}$ G. C. Ghirardi, C. Omero, and A. Rimini, "Stochastic interpretation of 
quantum mechanics-Critical review," Riv. Nuovo Cimento 1, 1-34 (1978).

${ }^{80}$ M. S. Wang and W. K. Liang, "Comment on repeated measurements in stochastic mechanics," Phys. Rev. D 48, 1875-1877 (1993).

${ }^{81}$ R. D. Astumian and P. Hänggi, "Brownian motors," Phys. Today 55 (11),
33-39 (2002); P. Reimann and P. Hänggi, "Quantum features of Brownian motors and stochastic resonance," Chaos 8, 629-642 (1998); P. Reimann, M. Grifoni, and P. Hänggi, "Quantum ratchets," Phys. Rev. Lett. 79, 10-13 (1997). 